\title{
Mechanisms responsible for nilotinib resistance in human chronic myeloid leukemia cells and reversal of resistance
}

\author{
Aylin Camgoz ${ }^{1}$, Emel Basak Gencer ${ }^{1}$, Ali Ugur Ural ${ }^{2} \&$ Yusuf Baran $^{1}$ \\ ${ }^{1}$ Department of Molecular Biology and Genetics, Faculty of Science, Izmir Institute of Technology, Izmir, Turkey \\ and ${ }^{2}$ Department of Hematology, Bayındır Hospital, Ankara, Turkey
}

\begin{abstract}
Multidrug resistance remains a significant obstacle to successful chemotherapy. The ability to determine the possible resistance mechanisms and surmount the resistance is likely to improve chemotherapy. Nilotinib is a very effective drug in the treatment of imatinib-sensitive or -resistant patients. Although very successful hematologic and cytogenetic responses have been obtained in nilotinib-treated patients, in recent years cases showing resistance to nilotinib have been observed. We aimed to examine the mechanisms underlying nilotinib resistance and to provide new targets for the treatment of chronic myeloid leukemia (CML). There was an up-regulation of antiapoptotic $\mathrm{BCR} / \mathrm{ABL}, \mathrm{GCS}$ and SK-1 genes and MRP1 transporter gene and down-regulation of apoptotic Bax and CerS1 genes in nilotinibresistant cells. There was no mutation in the nilotinib-binding region of $B C R / A B L$ in resistant cells. Inhibiton of GCS and SK-1 restored nilotinib sensitivity. Targeting the proteins that are involved in nilotinib resistance in addition to the inhibition of $\mathrm{BCR} / \mathrm{ABL}$ could be a better method of treatment in CML.
\end{abstract}

Keywords: Chronic myeloid leukemia, nilotinib, multidrug resistance, reversal of resistance

\section{Introduction}

Chronic myeloid leukemia (CML) is a disease of hematopoietic stem cells, arising from a reciprocal translocation between the long arms of chromosomes 9 and 22 known as the Philadelphia chromosome [1]. This translocation causes a juxtaposition of the ABL gene from chromosome 9 and the BCR gene from chromosome 22 resulting in generation of the $\mathrm{BCR} / \mathrm{ABL}$ fusion gene that encodes the BCR/ABL oncoprotein, which has constitutive oncogenic tyrosine kinase activity and undergoes cytoplasmic relocalization [2]. For many years CML was treated with imanitib, which is a selective tyrosine kinase inhibitor, for first-line therapy. Imatinib was the first tyrosine kinase inhibitor to show a strong activity in chronic and accelerated phases against BCR/ABL, while it is less effective in the blast phase of CML [3]. Its continuous administration was associated with the development of resistance, especially in the advanced phase or blast crisis [4]. In order to overcome imatinib resistance, more potent second-generation ABL kinase inhibitors were developed and have been used in clinical practice $[5,6]$.

Nilotinib is one of the most commonly used secondgeneration tyrosine kinase inhibitors, and is a very effective drug in the treatment of sensitive or imatinib-resistant patients in the clinic [5]. Nilotinib was methodically and rationally designed to create a better topological fit in the $\mathrm{ABL}$ kinase domain of $\mathrm{BCR} / \mathrm{ABL}$, resulting in enhanced $\mathrm{BCR} / \mathrm{ABL}$ inhibition. It is an aminopyrimidine derivative of imatinib, structurally changed to eliminate two energetically unfavorable hydrogen bonds with replacement of the $N$-methylpiperazine ring of imatinib by a trifluoromethylsubstituted phenyl group $[7,8]$. Unlike imatinib, nilotinib binds not only to the inactive conformation of ABL but also to the active conformation of ABL. On the other hand, unlike imatinib, nilotinib does not inhibit Src kinases [7].

Although very high hematologic and cytogenetic responses have been obtained in nilotinib-treated patients, recent resistance observed in patients is a problem $[9,10]$. Multidrug resistance mechanisms are responsible for the overall poor efficacy of chemotherapy in CML [11].

The main objectives of the present study were first to determine the responsible mechanisms underlying resistance to nilotinib and to increase the sensitivity of cells resistant to nilotinib in order to define new therapeutic targets for more effective treatment of CML.

\section{Materials and methods}

\section{Reagents}

Nilotinib was a gift from Novartis (Switzerland). It was dissolved in dimethylsulfoxide (DMSO) and $10 \mathrm{mM}$ stock solution was prepared and stored at $-20^{\circ} \mathrm{C}$. The final concentration of DMSO did not exceed more than $0.1 \%$ in

Correspondence: Assoc. Prof. Yusuf Baran, Izmir Institute of Technology, Faculty of Science, Department of Molecular Biology and Genetics, 35430 Urla, Izmir, Turkey. Tel: +90-232-7507515. Fax: +90-232-7507509. E-mail: yusufbaran@iyte.edu.tr, iytecancer@gmail.com 
culture. Primers were obtained from Eurofins (Germany). RPMI-1640, heat-inactivated fetal bovine serum (FBS), penicillin-streptomycin and trypsin-ethylenediaminetetraacetic acid (EDTA) were obtained from Biological Industries (Israel). An RNA isolation kit was obtained from Macherey-Nagel (Bethelem, PA). A DNA Gel Extraction Kit, Long PCR Enzyme Mix and Taq DNA Polymerase were obtained from Fermentas (Glen Burnie, MD). A dNTP set and DNA ladder were obtained from AMRESCO (Solon, $\mathrm{OH})$. A caspase- 3 colorimetric assay kit was obtained from BioVision (Mountain View, CA). An APO LOGIX JC-1 mitochondrial membrane potential detection kit was obtained from Cell Technology (Mountain View, CA). Bradford dye, Coomassie Blue, bovine serum albumin (BSA), trypan blue solution, $\beta$-mercaptoethanol, DMSO and agarose were all obtained from Sigma (St Louis, MO).

\section{Cell lines and culture conditions}

K562 human CML cells were obtained from the German Collection of Microorganisms and Cell Cultures (Germany). The cells were cultured in RPMI-1640 growth medium containing $15 \%$ fetal bovine serum and $1 \%$ penicillin-streptomycin at $37^{\circ} \mathrm{C}$ in $5 \% \mathrm{CO}_{2}$. Medium was refreshed every 3 days.

\section{Generation of nilotinib-resistant K562 cells}

The generation of resistant sub-lines was carried out in our research laboratory as described previously [12]. Briefly, K562 human CML parental cells were maintained in liquid cultures and exposed to stepwise increasing concentrations of nilotinib, starting with a concentration of $1 \mathrm{nM}$. As a final dose, $50 \mathrm{nM}$ nilotinib was applied to cells, and subpopulations of cells that were able to grow in the presence of $50 \mathrm{nM}$ nilotinib were referred to as K562/NIL-50.

\section{Measurement of changes in caspase- 3 activity}

A caspase-3 colorimetric assay kit (BioVision, Mountain View, CA) was used for the detection of caspase-3 activity. The activity assay was performed as described by the manufacturer. Briefly, $1 \times 10^{6}$ cells were seeded in a six-well plate in $2 \mathrm{~mL}$ growth medium in the absence or presence of increasing concentrations of nilotinib for $72 \mathrm{~h}$. Then, drug treated and untreated cells were collected by centrifugation in a Falcon tube at $1000 \mathrm{rpm}$ for $10 \mathrm{~min}$. The supernatant was gently removed and discarded while the cell pellet was lysed by the addition of $100 \mu \mathrm{L}$ of cold lysis buffer for each sample. The cell lysate was incubated on ice for $10 \mathrm{~min}$ before centrifugation at $14000 \mathrm{rpm}$ for $1 \mathrm{~min}$. Then, the supernatant was transferred to a new microcentrifuge tube. The enzymatic reaction for caspase activity was carried out in a 96-well flat bottom microplate that could be read with a microplate reader. For each reaction, $20 \mu \mathrm{L}$ of assay buffer $(5 \times), 25 \mu \mathrm{L}$ of sample, $50 \mu \mathrm{L}$ of sterilized water and $5 \mu \mathrm{L}$ of caspase- 3 colorimetric substrate (DEVD-pNA) were mixed and incubated for $2 \mathrm{~h}$ at $37^{\circ} \mathrm{C}$. The samples were read under a $405 \mathrm{~nm}$ wavelength by an enzyme linked immunosorbent assay (ELISA) reader (Multiskan Spectrum; Thermo Electron Corporation, Finland). After measuring protein concentrations by Bradford assay, enzyme activity levels were normalized to protein concentrations.

\section{Detection of loss of mitochondrial membrane potential}

The mitochondrion has a crucial role in the induction of intrinsic apoptosis via the loss of mitochondrial membrane potential (MMP). During this process, the electrochemical gradient across the mitochondrial membrane collapses. The reason for this collapse is thought to be the formation of pores in the mitochondria by dimerized Bax or activated Bid, Bak or Bad proteins. Activation of these pro-apoptotic proteins causes the release of cytochrome c into the cytoplasm. An APO LOGIX JC-1 Assay Kit (Cell Technology) was used to measure the loss of mitochondrial membrane potential in both K562 and K562/NIL-50 cells as described by the manufacturer.

Briefly, cells that had been induced to undergo apoptosis were collected by centrifugation at $1000 \mathrm{rpm}$ for $10 \mathrm{~min}$. Supernatants were removed, and $500 \mu \mathrm{L}$ of JC-1 dye (1\%) was added onto the pellets. After incubation of cells for $15 \mathrm{~min}$ at $37^{\circ} \mathrm{C}$ in $5 \% \mathrm{CO}_{2}$, they were centrifuged at $1000 \mathrm{rpm}$ for $5 \mathrm{~min}$. Then, $2 \mathrm{~mL}$ of assay buffer was added onto the pellets, and they were centrifuged for $5 \mathrm{~min}$ at $1000 \mathrm{rpm}$. All pellets were resuspended with $500 \mu \mathrm{L}$ assay buffer, and $150 \mu \mathrm{L}$ from each of them was added into black 96-well plates in triplicate. The aggregate red form has absorption/ emission maxima of 585/590 nm, and the green monomeric form has absorption/emission maxima of $510 / 527 \mathrm{~nm}$. The plate was read in these wavelengths by fluorescence ELISA reader (Varioskan Spectrum; Thermo Scientific, Finland). Finally, green/red $(510 / 585)$ values were calculated to determine the changes in MMP.

\section{Nucleotide sequence analyses of ATP binding site of ABL kinase domain in K562 and K562/NIL-50 cells}

To determine whether a point mutation in the BCR/ABL adenosine triphosphate (ATP)-binding domain was responsible for the resistance to nilotinib in K562/NIL-50 cells, the cDNA portion corresponding to the entire ABL kinase domain was sequenced. Total RNAs, isolated from K562 and K562/NIL-50 cells, were converted to cDNA by reverse transcriptase enzyme. Then, to amplify the ABL kinase domain of the BCR/ABL allele with forward primer BCR-F $\left(5^{\prime}\right.$-TGACCAACTCGTGTGTGAAACTC- $\left.3^{\prime}\right)$ and reverse primer ABL-R (5'-TCCACTTCGTCTGAGATACTGGATT-3'), a long polymerase chain reaction (PCR) method was used. ABL-F (5'-CGCAACAAGCCCACTGTCT-3') as forward primer and ABLkinase-R as reverse primer were used for a second-stage PCR. After that, the final PCR products were run on a $1 \%$ agarose gel at $90 \mathrm{~V}$ for $1 \mathrm{~h}$ and the ABL band was isolated from the gel by using a DNA Gel Extraction Kit as described by the manufacturer. The entire kinase domain was sequenced in the forward and reverse directions (Applied Biosystems 3130xl). Finally, the region including 863 bases was compared to the c-ABL known sequence (Gene Bank accession number: M14752).

\section{Total RNA isolation and reverse transcriptase-PCR}

The expression levels of ceramide synthase genes (CerS1-6), sphingosine kinase-1 (SK-1) gene and glucosyl ceramide synthase (GCS) gene were examined in K562 and K562/ NIL-50 cells. Total cellular RNAs were isolated using the RNA isolation kit (Macherey-Nagel). The recovered RNA con- 
centration was measured by Nanodrop ND-1000 (260/280 and 260/230 ratios). One microgram of total RNA was reverse transcribed into cDNA using reverse transcriptase enzyme (Moloney Murine Leukemia Virus Reverse Transcriptase; Fermentas). After 60 min incubation at $42^{\circ} \mathrm{C}$, the reactions were stopped at $70^{\circ} \mathrm{C}$ for $10 \mathrm{~min}$. The resulting total cDNA was used in a PCR to measure the mRNA levels of CerS1-6, SK-1, GCS, apoptosis genes, transporter genes, $\mathrm{BCR} / \mathrm{ABL}$ and $\beta$-actin as an internal positive control. Products of the PCR reactions were visualized under ultraviolet (UV) light after running them on $2 \%$ agarose gel electrophoresis including ethidium bromide at $90 \mathrm{~V}$ for $1 \mathrm{~h}$. Primer sequences are shown in Table I.

\section{Results}

\section{Involvement of mitochodria and caspase-3 activity in nilotinib resistance}

As we demonstrated previously, K562/NIL-50 cells showed more than 10-fold resistance to nilotinib as compared to the parental sensitive counterpart. The inhibitory concentration value $\left(\mathrm{IC}_{50}\right)$ of nilotinib that inhibits growth by $50 \%$ in these cells was $35 \mathrm{nM}$ and $386.5 \mathrm{nM}$ for K562 and K562/NIL-50 cells, respectively [12].

In order to demonstrate the role of apoptotic cell death in nilotinib resistance, we performed apoptotic assays to detect the loss of mitochondrial membrane potential and changes in caspase-3 activity.

Table I. Primer sequences.

\begin{tabular}{|c|c|}
\hline$\beta$-Actin-forward & $5^{\prime}$-CAGAGCAAGAGAGGCATCCT-3' \\
\hline$\beta$-Actin-reverse & 5'-TTGAAGGTCTCAAACATGAT-3' \\
\hline GCS-forward & 5'-ATGACAGAAAAAGTA-3' \\
\hline GCS-reverse & 5'-GGACACCCCTGAGTG-3' \\
\hline SK-1-forward & 5'-CCGACGAGGACTTTGTGCTAAT-3' \\
\hline SK-1-reverse & 5'-GCCTGTCCCCCCAAAGCATAAC-3' \\
\hline CerS1-forward & 5'-CTATACATGGACACCTGGCGCAA-3' \\
\hline CerS1-reverse & 5'-TCAGAAGCGCTTGTCCTTCACCA-3' \\
\hline CerS2-forward & 5'-GCTGGAGATTCACAT-3' \\
\hline CerS2-reverse & 5'-GAAGACGATGAAGAT-3' \\
\hline CerS4-forward & 5'-TGCTGTCCAGTTTCAACGAG-3' \\
\hline CerS4-reverse & 5'-GAGGAAGTGTTTCTCCAGCG-3' \\
\hline CerS5-forward & 5'-TCCTCAATGGCCTGCTGCTG-3' \\
\hline CerS5-reverse & 5'-CCCGGCAATGAAACTCACGC-3' \\
\hline CerS6-forward & 5'-CTCCCGCACAATGTCACCTG-3' \\
\hline CerS6-reverse & 5'-TGGCTTCTCCTGATTGCGTC-3' \\
\hline Bax-forward & 5'-ACCAAGAAGCTGAGCGAGTGT-3' \\
\hline Bax-reverse & 5'-ACAAACATGGTCACGGTCTGC-3' \\
\hline Bcl-xL-forward & 5'-GGAGCTGGTGGTTGACTTTCT-3' \\
\hline Bcl-xL-reverse & 5'-CCGGAAGGTTCATTCACTACT-3' \\
\hline Caspase-3-reverse & 5'-GGTTAACCCGGGTAAGAATGTGCA-3' \\
\hline Caspase-3-forward & 5'-CTCGGTCTGGTACAGATGTCGATG-3' \\
\hline BcL-2-forward & 5'-AGATGTCCAGCCAGCTGCACCTGAC-3' \\
\hline BcL-2-reverse & 5'-AGATAGGCACCCAGGGTGATGCAAGCTT-3' \\
\hline LRP-forward & 5'-CGCTGCTTGATTTTGAGGAT-3' \\
\hline LRP-reverse & 5'-CGAGAATCACGCAGTAGTTG-3' \\
\hline MRP1-forward & 5'-TAGAGGACTTCGTGTCAGCC-3' \\
\hline MRP1-reverse & 5'-GTCCATGATGGTGTTGAGCC-3' \\
\hline MDR1-forward & 5'-TACAGTGGAATTGGTGCTGGG-3' \\
\hline MDR1-reverse & 5'-CCCAGTGAAAAAATGTTGCCA-3' \\
\hline BCRP-forward & 5'-TACAGTTCTCAGCAGCTCTTCG-3' \\
\hline BCRP-reverse & 5'-CAACTTGAAGATGGAATATCGAG-3' \\
\hline $\begin{array}{l}\text { BCR/ABL, } \\
\text { B2B-forward }\end{array}$ & 5' -ACAGAATTCGCTGACCATCAATAAG-3' \\
\hline $\begin{array}{l}\text { BCR/ABL, } \\
\text { CA3-reverse }\end{array}$ & 5' -TGTTGACTGGCGTGATGTAGTTGCTTGG-3' \\
\hline
\end{tabular}

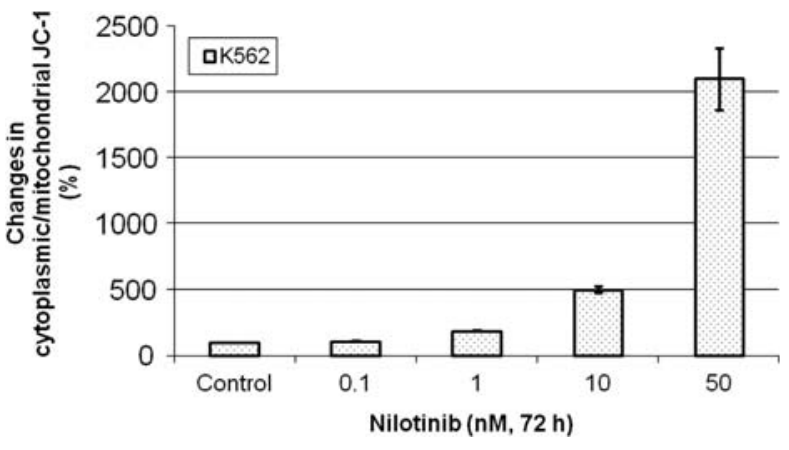

Figure 1. Percent changes in loss of MMP in K562 cells treated with nilotinib. Error bars represent standard deviations, and when not seen, they are smaller than the thickness of the lines on the graphs. MMP assays were performed using triplicate samples in at least three independent experiments. Statistical significance was determined using two-way analysis of variance, and $p<0.05$ was considered significant.

Treatment with $10 \mathrm{nM}$ nilotinib for $72 \mathrm{~h}$ caused a significant loss of MMP (about five-fold), as measured by increased accumulation of the cytoplasmic monomeric form of JC-1, in parental K562 (Figure 1), but not in resistant K562/NIL-50 cells (Figure 2). Moreover, there was only a 1.21-fold increase in loss of MMP in K562/NIL-50 cells in response to $50 \mathrm{nM}$ nilotinib, while the same concentration of nilotinib induced a 20.9-fold increased loss of MMP in K562 cells.

The activation of pro-caspase-3 in parental and resistant K562 cells was measured using the caspase- 3 activity assay. Treatment of K562 and K562/NIL-50 cells for $72 \mathrm{~h}$ with $10 \mathrm{nM}$ nilotinib resulted in 1.24- (Figure 3) and 1.06-fold (Figure 4) increases in caspase- 3 activity. In parallel with these results, $50 \mathrm{nM}$ nilotinib increased caspase- 3 activity by 1.79 - and 1.30-fold in K562 and K562/NIL-50 cells, respectively. Thus, these data confirmed that K562/NIL-50 cells exert significant resistance to nilotinib-induced loss of MMP and caspase-3 activation.

\section{Expression levels of $B C R / A B L$ in $\mathrm{K} 562$ and K562/NIL-50 cells}

Epression levels of BCR/ABL were determined in K562 and K562/NIL-50 cells by reverse transcriptase (RT)-PCR. Indeed, the data revealed that there was a significant increase in

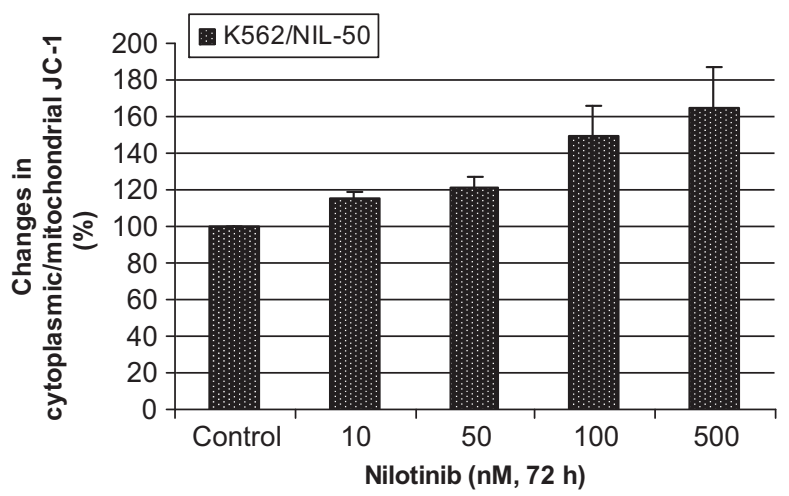

Figure 2. Percent changes in loss of MMP in K562/NIL-50 cells treated with nilotinib. Error bars represent standard deviations, and when not seen, they are smaller than the thickness of the lines on the graphs. MMP assays were performed in at least three independent experiments. Statistical significance was determined using two-way analysis of variance, and $p<0.05$ was considered significant. 


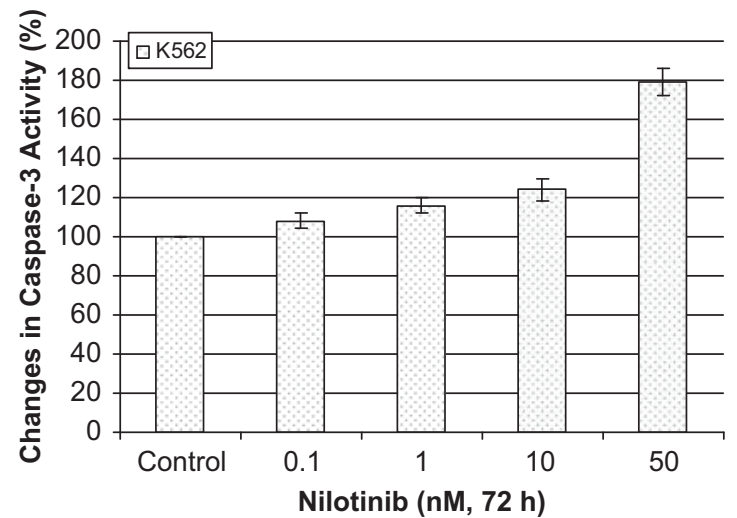

Figure 3. Fold changes in caspase-3 activity in response to nilotinib in K562 cells. Error bars represent standard deviations, and when not seen, they are smaller than the thickness of the lines on the graphs. Caspase-3 activity assays were performed in at least three independent experiments. Statistical significance was determined using two-way analysis of variance, and $p<0.05$ was considered significant.

expression levels of BCR/ABL in K562/NIL-50 cells as compared to their parental sensitive counterparts [Figure 5(A)]. Quantification analysis of BCR/ABL gene expression was conducted using the Quantitivel program, and the results showed that there was 3.85 -fold increase in expression of the $\mathrm{BCR} / \mathrm{ABL}$ gene in K562/NIL-50 cells, as compared to parental K562 cells [Figure 5(B)].

\section{Sequence analysis in nilotinib-binding site} of $A B L$ kinase domain in sensitive and resistant cells

In order to determine whether mutations on the ABL kinase domain affect the binding efficiency of nilotinib, sequence analyses of the ABL kinase region were examined in K562 and K562/NIL-50 cells. The data revealed that there were no detectable mutations at this region on $\mathrm{BCR} / \mathrm{ABL}$ in K562/NIL-50 cells (Figure 6).

\section{Expression levels of apoptosis related genes in K562 and K562/NIL-50 cells}

Expression levels of Bcl-2, Bcl-xL, Caspase-3 and Bax apoptosis regulating genes were analyzed in K562 and K562/ NIL-50 cells by RT-PCR (Figure 7). There were decreases

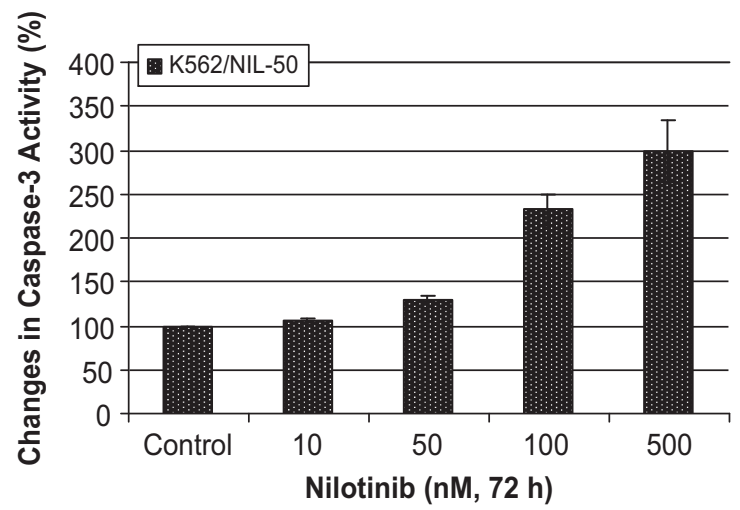

Figure 4. Fold changes in caspase-3 activity in response to nilotinib in K562/NIL-50 cells. Error bars represent standard deviations, and when not seen, they are smaller than the thickness of the lines on the graphs. Caspase- 3 activity assays were performed in at least three independent experiments. Statistical significance was determined using two-way analysis of variance, and $p<0.05$ was considered significant.
(A)
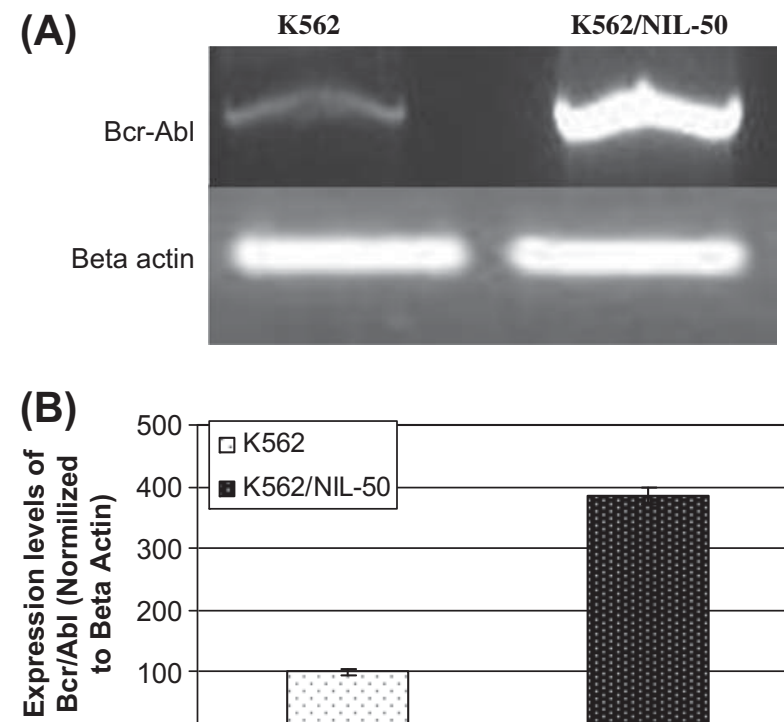

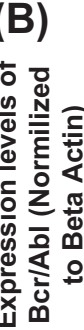

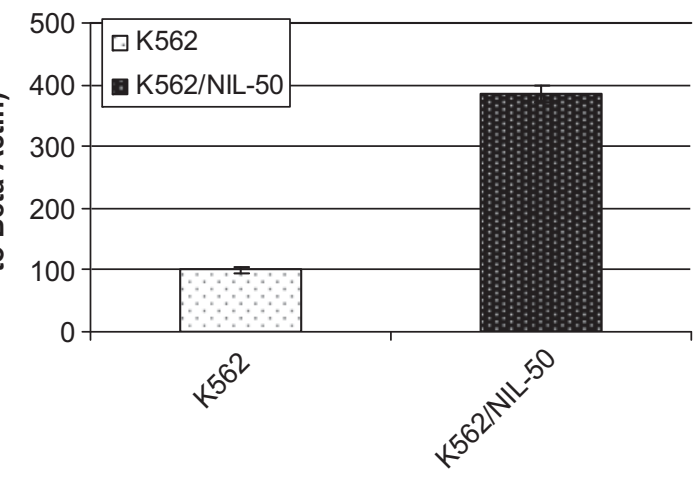

Figure 5. Expression analyses of BCR/ABL in parental and resistant CML cells by RT-PCR (A). Quantification of expression analysis of BCR/ ABL gene in K562 and K562/NIL-50 cells. Error bars represent standard deviations (B). Expression analyses were performed in at least three independent experiments.

in expression levels of Bax gene in K562/NIL-50 cells as compared to the parental sensitive counterparts. However, there were no changes in expression levels of Bcl-2, Bcl-xL and Caspase-3 genes. Quantification analysis of the expression levels of genes was conducted using the Quantitivel program. The results demonstrated that there were $2 \%, 3 \%$ and $25 \%$ decreases in K562/NIL-50 cells in the expression of Bcl-2, Bcl-xL and Bax genes, respectively, as compared to parental counterpart cells (Figure 7).

\section{Expression levels of ceramide metabolizing genes in $\mathrm{K} 562$ and K562/NIL-50 cells}

In order to determine the involvement of ceramide metabolizing genes in nilotinib resistance, expression levels of GCS, SK-1 and CerS family genes were analyzed. The data showed that there were significant increases in expression levels of GCS and SK-1 genes in K562/NIL-50 cells when compared to parental sensitive counterparts, whereas there was no significant change in expression levels of CerS genes (Figure 8). There were 2.45- and 2.04-fold increases in expression levels of antiapoptotic GCS and SK-1 genes in K562/NIL-50 cells, respectively, as compared to parental sensitive cells (Figure 8).

\section{Expression levels of transport family genes in K562 and K562/NIL-50 cells}

In order to determine the role of transporter genes in nilotinib resistance, expression levels of MDR1, MRP1, BCRP and LRP genes were examined in K562 and K562/NIL-50 cells by RTPCR. The data showed that there were slight increases in expression levels of the MRP1 gene in K562/NIL-50 cells as compared to the parental sensitive counterparts (Figure 9). Interestingly, 


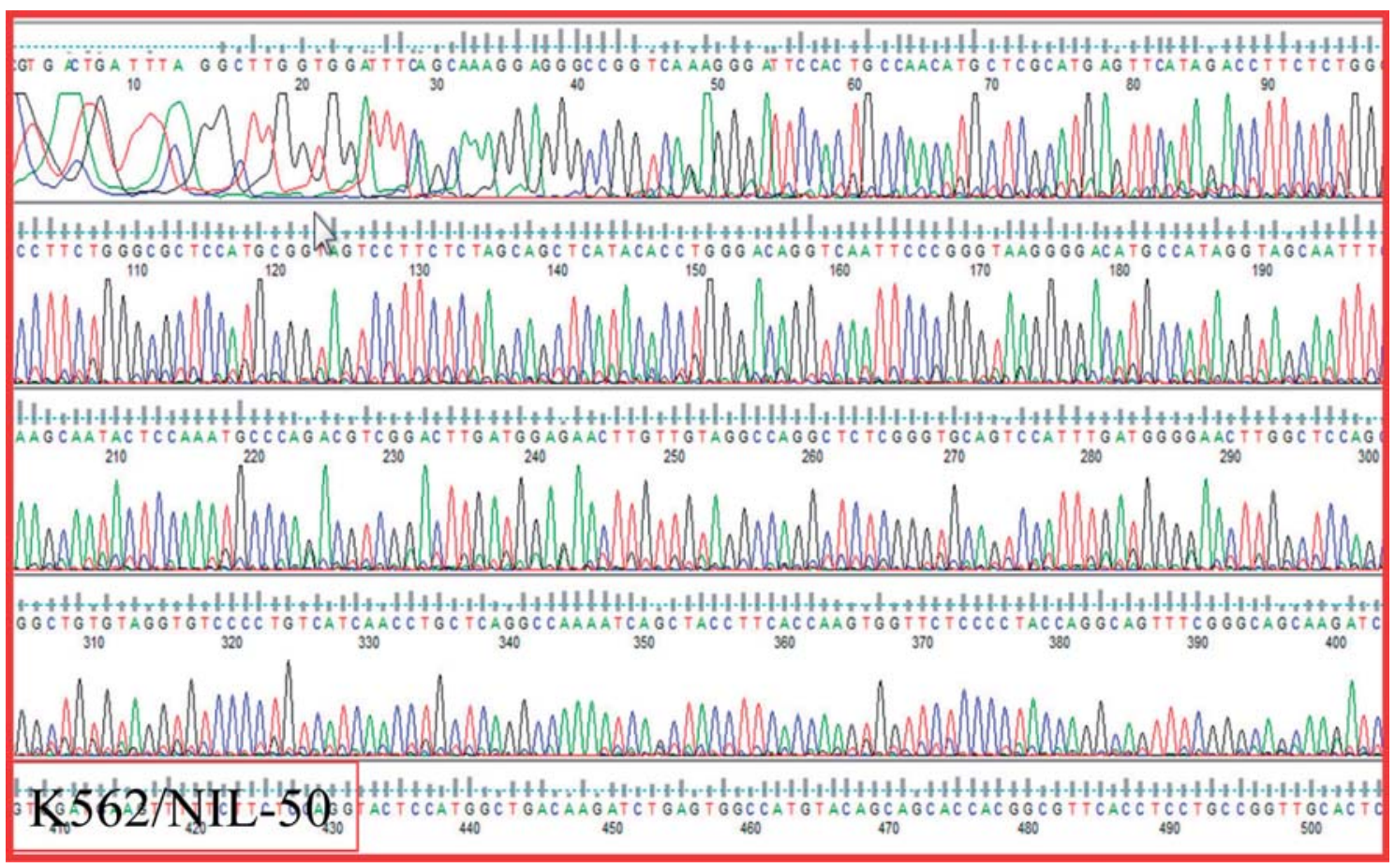

Figure 6. Nucleotide sequence analyses of ABL kinase domain of K562/NIL-50 cells.

MDR1, BCRP and LRP genes were down-regulated in K562/ NIL-50 cells using the Quantitivel program.

\section{Reversal of nilotinib resistance through inhibition of ceramide metabolizing genes in combination with nilotinib in K562/NIL-50 cells}

In order to determine whether nilotinib resistance can be reversed by targeting ceramide metabolizing genes in combination with nilotinib, cell proliferation, changes in caspase-3 enzyme activity, and loss of MMP were examined. As shown in Figure 10, the GCS inhibitor, PDMP, or SK-1 inhibitor together with increasing concentrations of nilotinib decreased proliferation of K562/NIL-50 cells significantly in a dose-dependent manner [Figures 10(A) and 10(B)]. In order to confirm reversal of nilotinib resistance, changes in caspase-3 enzyme activity were also investigated. While the data showed that nilotinib in combination with

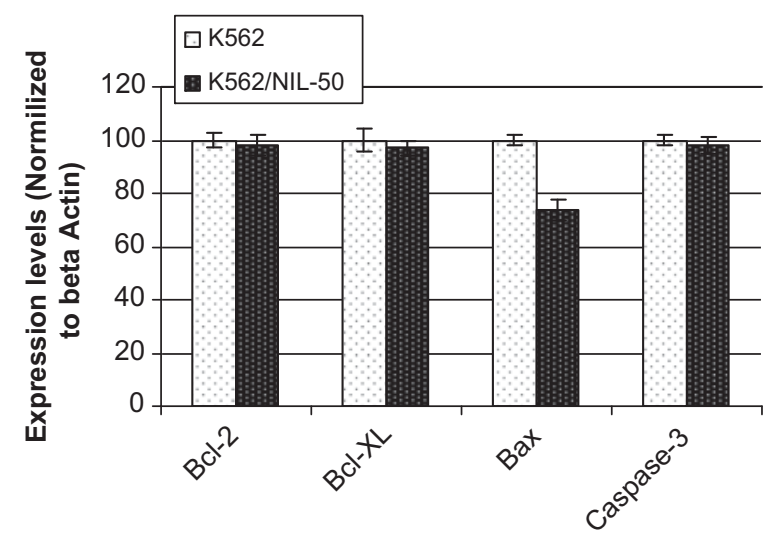

Figure 7. Expression levels of Bcl-2, Bcl-xL, Caspase- 3 and Bax apoptosis regulating genes in K562 and K562/NIL-50 cells. Expression analyses were performed in at least three independent experiments. Error bars represent standard deviations.
PDMP increased caspase-3 enzyme activity dramatically as compared to nilotinib or PDMP alone, there was a moderate increase in caspase-3 enzyme activity when cells were treated by nilotinib in combination with SK-1 inhibitor (Figure 11). Moreover, there were synergistic effects of PDMP and nilotinib in terms of inducing apoptosis, which was shown by an increase in loss of MMP (Figure 12).

Similar results were obtained with the combination of nilotinib and SK-1 inhibitor. The same concentrations of nilotinib (20 and $50 \mathrm{nM}$ ) were applied to cells together with SK-1 inhibitor, which triggered a significant increase in loss of MMP (Figure 12).

\section{Discussion}

CML has been a model disease since its discovery, because CML was the first neoplasm found to be associated with a chromosomal translocation, known as the Philadelphia chromosome. This feature provided the means to study the molecular basis of CML, with the aim of leading to targeted and more effective treatment for CML. Subsequently, the availability of molecular targeted therapy has profoundly changed the management of CML and defined general ideas regarding cancer treatment [13].

Imatinib was the first tyrosine kinase inhibitor to show strong activity in the chronic and accelerated phases, while it is less effective in the blast phase of CML [3]. Unfortunately, the emergence of resistance is a major problem in CML treatment $[14,15]$. The second-generation tyrosine kinase inhibitors, including nilotinib, were designed to overcome this resistance. Nilotinib is now used as a first-line treatment, with approval by the Food and Drug Adminsitration (FDA) since July 2010 . However, there have been recently observed cases of nilotinib resistance in patients with CML. 


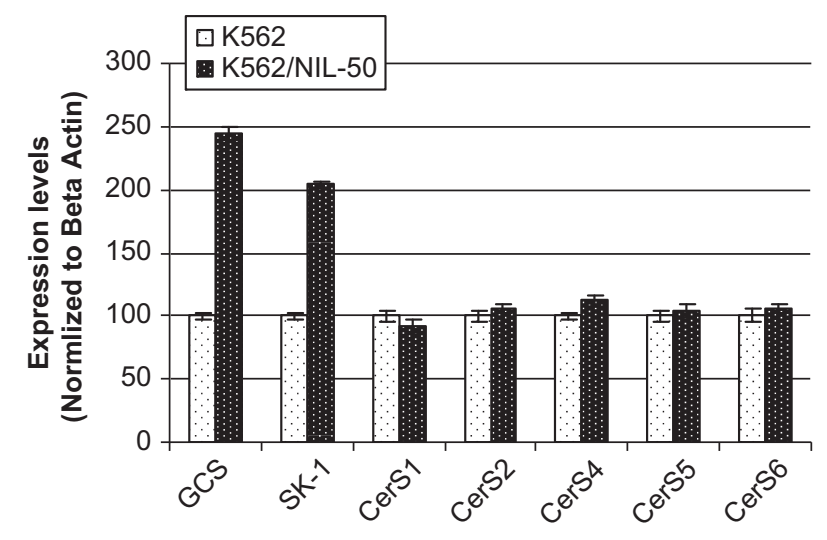

Figure 8. Expression levels of ceramide metabolizing genes in K562 and K562/NIL-50 cells. Expression analyses were performed in at least three independent experiments. Error bars represent standard deviations.

In this study, the possible molecular mechanisms of nilotinib resistance were investigated in K562/NIL-50 CML cells, and increasing nilotinib sensitivity was examined by targeting SK-1 and GCS.

A similar approach was applied to obtain resistance in leukemic cells by our group and in some other previous studies $[16,17]$. Mahon and co-workers were able to develop nilotinib-resistant AR230, LAMA84 and K562 cells [18]. However, the cells they developed were only $20 \mathrm{nM}$ nilotinib-resistant cells. In our study we were able to generate $50 \mathrm{nM}$ nilotinibresistant K562 cells, and to our knowledge, K562/NIL-50 cells are the most nilotinib-resistant cells according to the current literature.

It has been well documented that the degree of BCR/ABL expression appears to be directly proportional to the level of drug resistance $[16,19]$. In the present study, confirming previous data, BCR/ABL was overexpressed significantly in K562/NIL-50 cells as compared to parental sensitive counterparts. Besides, Mahon et al. showed that resistance to nilotinib may be mediated by up-regulated expression of BCR/ABL [18].

The main mechanism responsible for the development of resistance in CML is related to the point mutations that prevent the binding of inhibitors. This resistance mechanism was due to selection of cells with mutated BCR/ABL in the imatinib-binding domain in various CML cell lines [20].

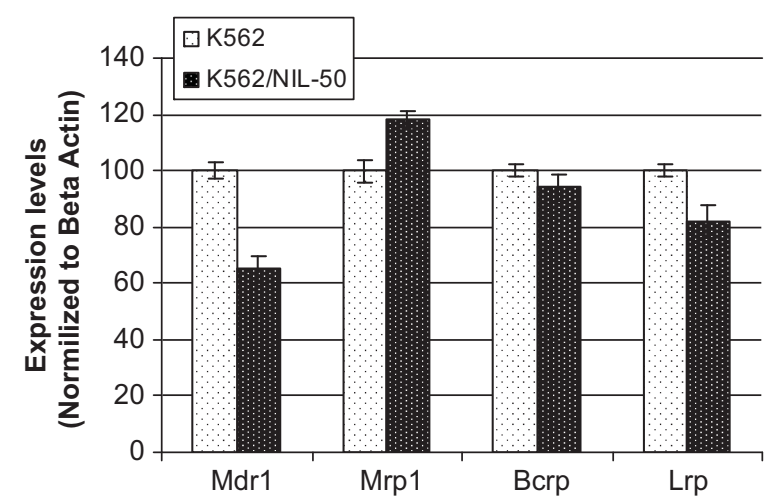

Figure 9. Expression levels of transporter genes in K562 and K562/ NIL-50 cells. Expression analyses were performed in at least three independent experiments. Error bars represent standard deviations.
An extensive search for the presence of all mutations in parental and resistant K562/NIL-50 cells using RT-PCR followed by direct sequencing failed to identify any of these mutations. These findings may show that resistance to nilotinib in these resistant human CML cells did not result from any mutation in the nilotinib-binding site of the ABL kinase domain.

On the other hand, BCR/ABL independent mechanisms have been reported as leading to resistance in various $\mathrm{CML}$ cells. Nilotinib resistance can be due to a failure to induce apoptosis. Signals inducing apoptosis may be blocked and/ or antiapoptotic gene overexpression can be observed in resistant cells. The Bcl-2 protein can block apoptosis induced by most chemotherapeutic agents [21]. Up-regulation of antiapoptotic Bcl-2 occurs by a Lyn-kinase-dependent mechanism [22]. Our results demosntrated that the apoptotic Bax gene was relatively down-regulated, while there were no changes in the expression levels of Bcl-2 and Bcl-xL. Bax is a pro-apoptotic molecule which is responsible for the formation of pores on the outer mitochondrial membrane, resulting in a loss of mitochondrial membrane potential which activates caspases $(-3,-7$ and -9$)$ and nucleases by cytochrome c release from the mitochondria [23]. These data are in agreement with our study which showed that in nilotinib-treated parental human CML cells, there were significant losses in MMP and increases in caspase-3 enzyme activity, resulting in a higher number of cells undergoing apoptosis. Thus, the pro-apoptotic effects of nilotinib may be explained by a rapid and sustained inhibition of BCR/ $\mathrm{ABL}$, leading to induction of mitochondria-dependent apoptosis in parental cells. At steady state conditions, decreases were detected in loss of MMP and in caspase-3 activity in resistant cells as compared to their parental sensitive counterparts. Mitochondrial membrane potential and caspase- 3 enzyme activity analyses revealed that although higher concentrations of nilotinib were applied, there was almost no loss of mitochondrial membrane potential and no increase in caspase-3 enzyme activity in resistant cells.

In addition, transporter-mediated tyrosine kinase inhibitor efflux has been implicated as a possible mechanism for resistance to imatinib [24,25]. However, there has been significant controversy in the field regarding the potential of $\mathrm{ABC}$ transporters to confer drug resistance. There are several studies showing that MDR1 and BCRP transporters are involved in nilotinib resistance as substrate or inhibitor $[26,27]$. One report described an interaction between nilotinib and BCRP, suggesting that nilotinib is a BCRP substrate [27], while another study showed that at higher concentrations, nilotinib reduced both MDR1 and BCRP activities [28]. Our results revealed that mRNA expression of MRP1 gene was up-regulated in K562/NIL-50 cells as compared to K562 cells, indicating that MRP1 overexpression may be a mechanism of nilotinib resistance. Although there are only a few reports regarding the role of MRP1 in drug resistance in the literature, Shen et al. showed that nilotinib reverses MRP7 mediated paclitaxel resistance at a very high concentration (5 $\mu \mathrm{M})$ [29].

Furthermore, we examined the role of bioactive sphingolipids in the regulation of nilotinib resistance in human K562 


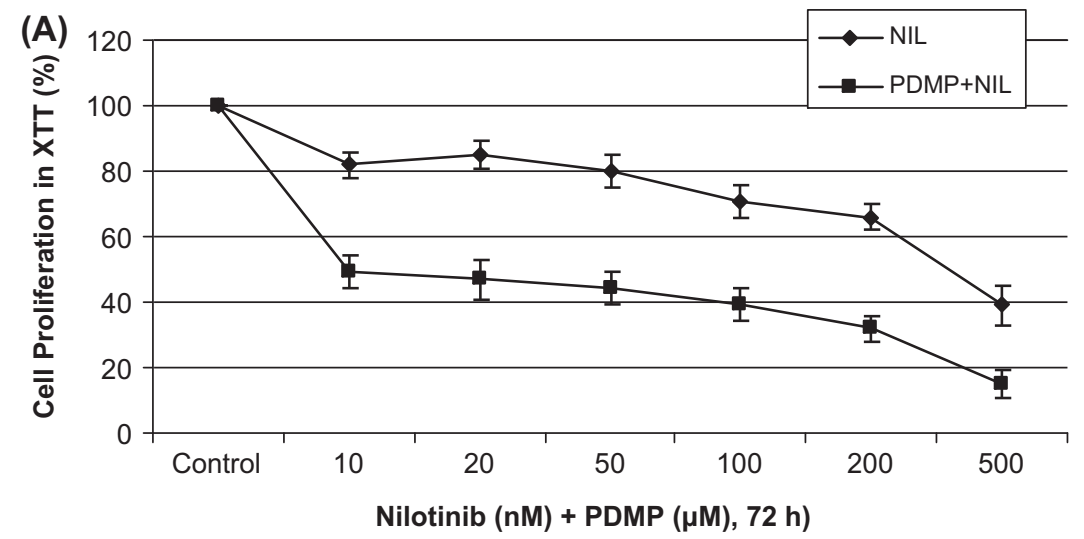

(B)

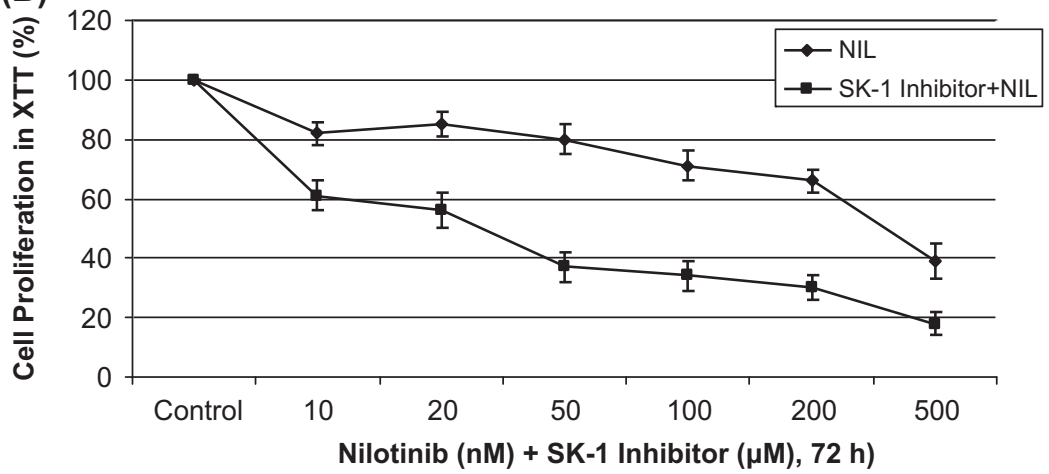

Figure 10. Cell proliferation in response to nilotinib alone and in combination with PDMP (A) and SK-1 inhibitor (B) in K562/NIL-50 cells. XTT assays were performed using triplicate samples in at least three independent experiments. Error bars represent standard deviations. Statistical significance was determined using two-way analysis of variance, and $p<0.05$ was considered significant.

cells, as another BCR/ABL independent resistance mechanism. The bioactive sphingolipid, ceramide, is involved in mediating antiproliferative responses via various different mechanisms in human cancer cells [30]. It has been well documented that treatment with some chemotherapeutic agents results in increased generation and/or accumulation of endogenous ceramide either via activation of a de novo pathway (CerS-6 genes), or by increased activity of SMases [30]. However, any role for nilotinib in inducing the generation of ceramide in human CML cells has not been previously described. Here, the data showed that there were significant increases in expression levels of GCS and SK-1 genes, which are responsible for converting apoptotic ceramides to antiapoptotic glucosyl ceramide and sphingosine-1 phosphate, respectively. There are several reports showing that SK-1 is overexpressed in distinct cancer types [31-34]. Besides, GCS overexpression has been observed in imatinibresistant human CML cells [35]. Another study supports the involvement of GCS in drug resistance, showing that overexpression of the GCS enzyme resulted in increased resistance to adriamycin in drug sensitive MCF-7 tumor cells [36]. In our study, we demonstrated that there were synergistic antiproliferative and apoptotic effects of nilotinib in combination with GCS or SK-1 inhibitors. A decrease in expression levels of CerS1 that selectively regulates the synthesis of $\mathrm{C}_{18}$-ceramide was observed, while CerS2, CerS4, CerS5 and

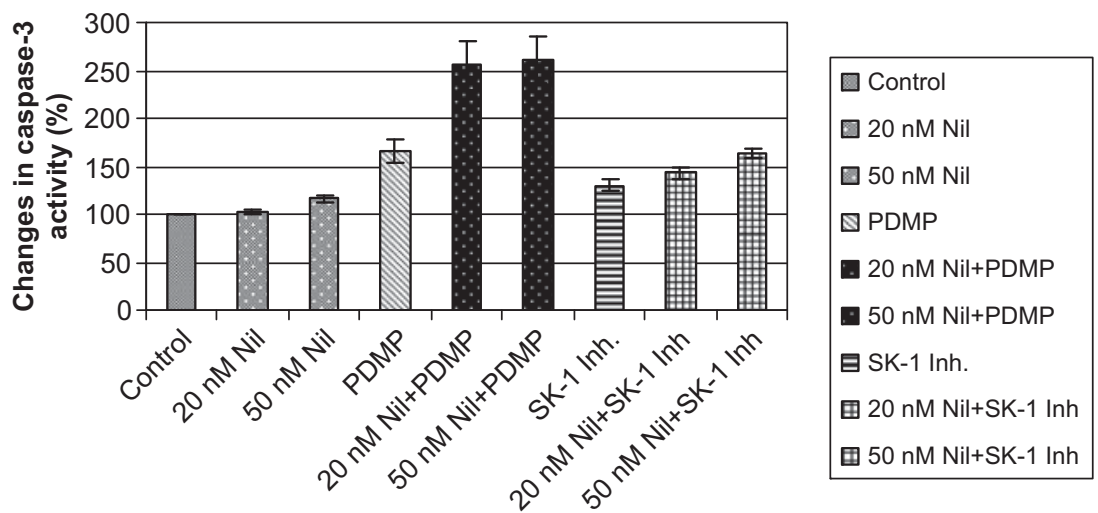

Figure 11. Fold changes in caspase-3 activity in response to nilotinib alone and in combination with PDMP and SK-1 inhibitor in K562/NIL-50 cells. Caspase-3 activity assays were performed in at least three independent experiments. Error bars represent standard deviations. Statistical significance was determined using two-way analysis of variance, and $p<0.05$ was considered significant. 


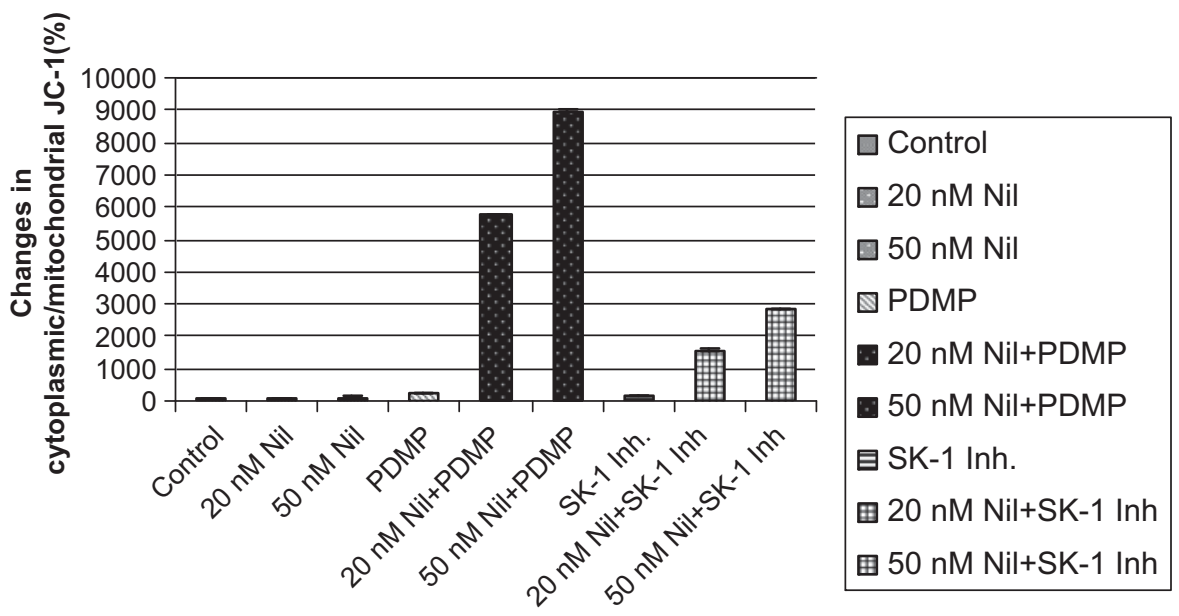

Figure 12. Percent changes in loss of MMP in response to nilotinib alone and in combination with PDMP and SK-1 in K562/NIL-50 cells. MMP assays were performed in at least three independent experiments. Error bars represent standard deviations. Statistical significance was determined using two-way analysis of variance, and $p<0.05$ was considered significant.

CerS6 were still up-regulated in K562/NIL-50 cells. These data are in agreement with a study showing that when CerS1 was overexpressed [37], imatinib resistant cells had increased sensitivity to imatinib, suggesting that up-regulation of ceramide generation might help to improve the response to imatinib. However, the overexpression of CerS2, CerS5 and CerS6 did not cause any change in imatinib resistant cells [33]. According to these results, a decrease in expression of CerS1 may also be responsible for nilotinib resistance.

In summary, we have developed cells resistant to nilotinib at very high concentrations, up to $50 \mathrm{nM}$. More importantly, these results show that ceramide metabolizing genes including GCS and SK-1 may be involved in the regulation of nilotinib resistance in K562 cells. A strong strategy for designing novel therapies for the treatment of CML could be targeting ceramide clearence genes together with inhibition of BCR/ABL.

\section{Acknowledgements}

This study was supported by TUBITAK Project number 107S317. We thank the Biotechnology and Bioengineering Center staff of Izmir Institute of Technology for their help and technical support.

Potential conflict of interest: Disclosure forms provided by the authors are available with the full text of this article at www.informahealthcare.com/lal.

\section{References}

[1] Druker BJ, O’Brien SG, Cortes J, et al. Chronic myelogenous leukemia. Hematology Am Soc Hematol Educ Program 2002: 111-135.

[2] Koca E, Haznedaroglu IC. Imatinib mesylate and the management of chronic myeloid leukemia (CML). Turk J Hematol 2005;2:161-172.

[3] Savage DG, Antman KH. Imatinib mesylate--a new oral targeted therapy. N Engl J Med 2002;346:683-693.

[4] Bonhoure E, Lauret A, Barnes DJ, et al. Sphingosine kinase-1 is a downstream regulator of imatinib-induced apoptosis in chronic myeloid leukemia cells. Leukemia 2008;22:971-979.
[5] Kantarjian H, Giles F, Wunderle L, et al. Nilotinib in imatinibresistant CML and Philadelphia chromosome-positive ALL. N Engl J Med 2006;354:2542-2551.

[6] Talpaz M, Shah NP, Kantarjian H, et al. Dasatinib in imatinibresistant Philadelphia chromosome-positive leukemias. N Engl J Med 2006;354:2531-2541.

[7] Weisberg E, Manley PW, Breitenstein W, et al. Characterization of AMN107, a selective inhibitor of native and mutant Bcr-Abl. Cancer Cell 2005;7:129-141.

[8] O'Hare T, Walters DK, Deininger MW, et al. AMN107: tightening the grip of imatinib. Cancer Cell 2005;7:117-119.

[9] Hughes T, Saglio G, Branford S, et al. Impact of baseline BCR-ABL mutations on response to nilotinib in patients with chronic myeloid leukemia in chronic phase. J Clin Oncol 2009;27:4204-4210.

[10] Saglio G, Kim DW, Issaragrisil S, et al. Nilotinib versus imatinib for newly diagnosed chronic myeloid leukemia. N Engl J Med 2010;362:2251-2259.

[11] Hochhaus A, Kreil S, Corbin AS, et al. Molecular and chromosomal mechanisms of resistance to imatinib (STI571) therapy. Leukemia 2002;16:2190-2196.

[12] Ceylan C, Camgoz A, Baran Y. Macromolecular changes in nilotinib resistant K562 cells; an in vitro study by Fourier transform infrared spectroscopy. Technol Cancer Res Treat 2012;11: 333-344.

[13] Hehlmann R, Berger U, Pfirrmann M, et al. Drug treatment is superior to allografting as first-line therapy in chronic myeloid leukemia. Blood 2007;109:4686-4692.

[14] Walz C, Sattler M. Novel targeted therapies to overcome imatinib mesylate resistance in chronic myeloid leukemia (CML). Crit Rev Oncol Hematol 2006;57:145-164.

[15] Deininger M. Resistance to imatinib: mechanisms and management. J Natl Compr Canc Netw 2005;3:757-768.

[16] Mahon FX, Deininger MW, Schultheis B, et al. Selection and characterization of BCR-ABL positive cell lines with differential sensitivity to the tyrosine kinase inhibitor STI571: diverse mechanisms of resistance. Blood 2000;96:1070-1079.

[17] Baran Y, Ural AU, Gunduz U. Mechanisms of cellular resistance to imatinib in human chronic myeloid leukemia cells. Hematology 2007;12:497-503.

[18] Mahon FX, Hayette S, Lagarde V, et al. Evidence that resistance to nilotinib may be due to BCR-ABL, Pgp, or Src kinase overexpression. Cancer Res 2008;68:9809-9816.

[19] Weisberg E, Griffin JD. Mechanism of resistance to the ABL tyrosine kinase inhibitor STI571 in BCR/ABL-transformed hematopoietic cell lines. Blood 2000;95:3498-3505.

[20] Wang Y, Cai D, Brendel C, et al. Adaptive secretion of granulocytemacrophage colony-stimulating factor (GM-CSF) mediates imatinib and nilotinib resistance in BCR/ABL + progenitors via JAK-2/STAT-5 pathway activation. Blood 2007;109:2147-2155.

[21] Reed JC. Bcl-2-family proteins and hematologic malignancies: history and future prospects. Blood 2008;111:3322-3330.

[22] Dai Y, Rahmani M, Corey SJ, et al. A Bcr/Abl-independent, Lyn-dependent form of imatinib mesylate (STI-571) resistance is 
associated with altered expression of Bcl-2. J Biol Chem 2004;279: 34227-34239.

[23] Zamzami N, Brenner C, Marzo I, et al. Subcellular and submitochondrial mode of action of Bcl-2-like oncoproteins. Oncogene 1998;16:2265-2282.

[24] Thomas DA, Faderl S, Cortes J, et al. Treatment of Philadelphia chromosome-positive acute lymphocytic leukemia with hyper-CVAD and imatinib mesylate. Blood 2004;103:4396-4407.

[25] Jiang X, Zhao Y, Smith C, et al. Chronic myeloid leukemia stem cells possess multiple unique features of resistance to BCR-ABL targeted therapies. Leukemia 2007;21:926-935.

[26] Dohse M, Scharenberg C, Shukla S, et al. Comparison of ATP-binding cassette transporter interactions with the tyrosine kinase inhibitors imatinib, nilotinib, and dasatinib. Drug Metab Dispos 2010;38:1371-1380.

[27] Brendel C, Scharenberg C, Dohse M, et al. Imatinib mesylate and nilotinib (AMN107) exhibit high-affinity interaction with ABCG2 on primitive hematopoietic stem cells. Leukemia 2007;21:1267-1275.

[28] Hegedus C, Truta-Feles K, Antalffy G, et al. Interaction of the EGFR inhibitors gefitinib, vandetanib, pelitinib and neratinib with the ABCG2 multidrug transporter: Implications for the emergence and reversal of cancer drug resistance. Biochem Pharmacol 2012;84: 260-267.

[29] Shen T, Kuang YH, Ashby CR, et al. Imatinib and nilotinib reverse multidrug resistance in cancer cells by inhibiting the efflux activity of the MRP7 (ABCC10). PLoS One 2009;4:e7520(1-9).
[30] Ogretmen B, Hannun YA. Biologically active sphingolipids in cancer pathogenesis and treatment. Nat Rev Cancer 2004;4:604-616.

[31] Sobue S, Iwasaki T, Sugisaki C, et al. Quantitative RT-PCR analysis of sphingolipid metabolic enzymes in acute leukemia and myelodysplastic syndromes. Leukemia 2006;20:2042-2046.

[32] French KJ, Schrecengost RS, Lee BD, et al. Discovery and evaluation of inhibitors of human sphingosine kinase. Cancer Res 2003;63:5962-5969.

[33] Li QF, Wu CT, Guo Q, et al. Sphingosine 1-phosphate induces Mcl-1 upregulation and protects multiple myeloma cells against apoptosis. Biochem Biophys Res Commun 2008;371:159-162.

[34] Baran Y, Salas A, Senkal CE, et al. Alterations of ceramide/ sphingosine 1-phosphate rheostat involved in the regulation of resistance to imatinib-induced apoptosis in K562 human chronic myeloid leukemia cells. J Biol Chem 2007;282:10922-10934.

[35] Baran Y, Bielawski J, Gunduz U, et al. Targeting glucosylceramide synthase sensitizes imatinib-resistant chronic myeloid leukemia cells via endogenous ceramide accumulation. J Cancer Res Clin Oncol 2011;137:1535-1544.

[36] Liu YY, Han TY, Giuliano AE, et al. Expression of glucosylceramide synthase, converting ceramide to glucosylceramide, confers adriamycin resistance in human breast cancer cells. J Biol Chem 1999;274:1140-1146.

[37] Venkataraman K, Futerman AH. Do longevity assurance genes containing Hox domains regulate cell development via ceramide synthesis? FEBS Lett 2002;528:3-4. 